\title{
Risk factors for the development of deformity in patients with spinal infection
}

\author{
Dushyanth Srinivasan, B.A., Samuel W. Terman, M.D., M.S., Mai Himedan, B.S., \\ Domenico Dugo, Frank La Marca, M.D., and Paul Park, M.D.
}

Department of Neurosurgery, University of Michigan, Ann Arbor, Michigan

\begin{abstract}
Object. Bacterial spinal infections are infrequent but may lead to significant morbidity and death. Apart from neurological complications, infections may also lead to bone destruction resulting in deformity of the spine. However, the incidence of spinal deformities and risk factors is not well characterized in the literature.

Methods. A retrospective cohort study was conducted using electronic medical records at a single institution. All patients were over 18 years of age and had a clinically and radiologically documented spinal infection that was treated surgically during the period 2006-2013. Infections were classified according to anatomical location including disc, bone, and/or epidural space. Deformities included kyphosis and/or subluxation. The authors analyzed risk factors for developing at least 1 deformity between the time of infection and operation using the Fisher exact test and chi-square test. Change in visual analog scale (VAS) scores preoperatively versus postoperatively was also analyzed using the paired t-test.

Results. The study included 48 patients. The most common types of spinal infections were osteomyelitis and discitis (31\%); osteomyelitis, discitis, and spinal epidural abscess (SEA; 27\%); SEA only (15\%); and osteomyelitis only $(13 \%)$. Overall, $21(44 \%)$ of 48 patients developed a spinal deformity. Anatomical location of infection (bone and/or disc and/or epidural space) was significantly associated with development of deformity ( $\mathrm{p}<0.001)$. In particular, patients with SEA had lower odds of deformity compared with patients without SEA (odds ratio $0.2,95 \%$ confidence interval $0.05-0.9 ; \mathrm{p}<0.001)$. No other factor was significantly associated with deformity. Pain measured by VAS score tended to improve by a mean of $1.7 \pm 2.7$ points $(\mathrm{p}<0.001)$ when comparing preoperative to postoperative scores.

Conclusions. In this cohort of patients, $44 \%$ developed at least 1 deformity, predominantly kyphosis. The only variable significantly associated with deformity was infection location. Patients with SEA alone demonstrated lower odds of developing a deformity compared with patients without SEA. Other analyzed variables, including age, body mass index, time from initial diagnosis to surgery, and comorbidities, were not found to be associated with development of deformity. Surgical intervention resulted in pain improvement.
\end{abstract}

(http://thejns.org/doi/abs/10.3171/2014.6.FOCUS14143)

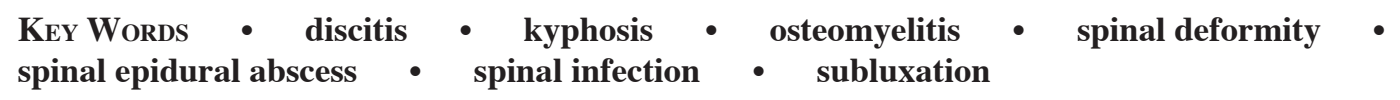

B ACTERIAL spinal infection can involve the intervertebral disc, bone, and/or epidural space. Incidence of spinal epidural abscess (SEA) has been reported to range from 0.2 to 1.2 cases per 10,000 hospital admissions. ${ }^{13}$ The incidence of vertebral osteomyelitis has been estimated to be 2.2 per 100,000 persons. ${ }^{3}$ Various risk factors for spinal infection have been reported, including intravenous drug abuse, diabetes, chronic alcohol abuse, immunosuppression, and trauma. ${ }^{11,13}$ Hematogenous dissemination is typically the origin of spinal infection, and onset of symptoms can be insidious, with back pain the most common complaint.

In addition to neurological deficits, spinal infection can result in instability and deformity necessitating the need for surgical stabilization. ${ }^{1,6,9,12}$ Although spinal in-

\footnotetext{
Abbreviations used in this paper: $\mathrm{BMI}=$ body mass index; $\mathrm{SEA}$ $=$ spinal epidural abscess; VAS $=$ visual analog scale.
}

fection causing deformity has been well documented, there are relatively few studies focusing on risk factors associated with the development of deformity. The purpose of this study was to assess multiple potential risk factors for the development of spinal deformity due to infection.

\section{Methods}

Study Design

Following approval from the University of Michigan Institutional Review Board, we performed a retrospective cohort study. ${ }^{14}$ Among patients surgically treated for a spinal infection, we determined the association between a variety of patient-centered demographic and medical factors with the occurrence of spinal deformity. We also sought to describe the absolute frequency of spinal infection among this population overall and in various subpopulations. 
Three potential sites of infection were categorized for each patient: disc, bone, and/or epidural space. Patients could thus be classified as having discitis, osteomyelitis, SEA, or any combination of the 3 categories.

\section{Patient Population}

We studied patients at least 18 years old, with at least 1 site of spinal infection diagnosed and surgically treated by a single surgeon (P.P.) at a single institution for the period 2006-2013. All patients who fit these criteria were included in the analysis. Surgical treatment involved instrumented fusion for cases involving deformity, while cases involving SEA alone were typically treated with laminectomy for debridement and decompression.

\section{Data Collection and Outcomes Assessment}

All data were collected from electronic medical records. Demographic information such as age at operation, sex, and body mass index (BMI) was collected. Relevant medical history, including prior spinal surgery, osteoarthritis, osteoporosis, hypertension, diabetes mellitus, coronary artery disease, and congestive heart failure, was determined, as documented by preoperative history and physical examination. Immediate preoperative creatinine level was recorded. Operative details such as the number of levels fused and microbiological results were determined from operative notes and consultation notes from the infectious disease department.

Spinal infection was defined as a clinical diagnosis incorporating history and physical examination and required radiological confirmation of osteomyelitis, discitis, or SEA. Our definition did not require microbiological confirmation.

Our primary outcome of interest was the occurrence of spinal deformity prior to surgical intervention. Spinal deformity was defined as a radiological diagnosis confirming changes in the sagittal or coronal plane consistent with kyphosis and subluxation of the vertebral column. When prior spinal imaging was available, significant kyphosis or subluxation was identified by a change from the baseline radiological studies (Fig. 1). In cases in which prior imaging was unavailable, determination of significant kyphosis or subluxation was made by radiologist and surgeon interpretations of the imaging, showing focal angulation or slippage (Figs. 2 and 3). Our secondary outcome of interest involved comparison of preoperative versus postoperative visual analog scale (VAS) scores for spine pain. Visual analog scale scores comprise an ordinal score from 1 (no pain) to 10 (worst imaginable pain). The VAS scores were obtained from patients at their preoperative visit and at their last follow-up visit.

\section{Statistical Analysis}

Descriptive statistics were used to characterize the patient cohort. Continuous variables were described using means \pm standard deviations, while categorical variables were described as absolute frequencies. To determine factors associated with spinal deformities, we used chi-square or Fisher exact tests as appropriate for categorical variables. Continuous variables were categorized

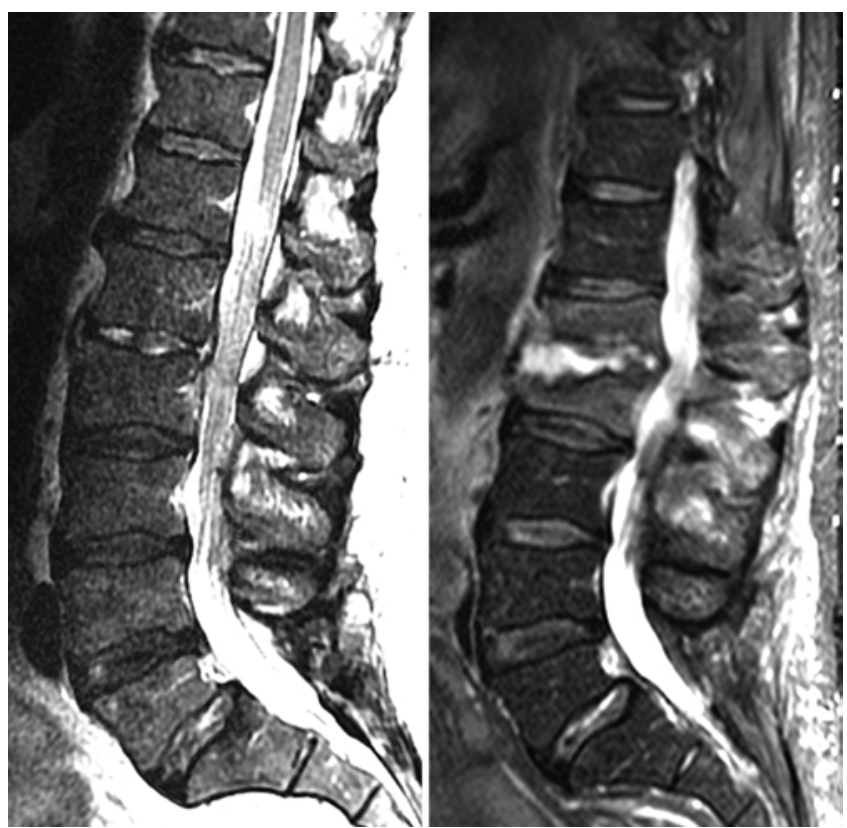

FIG. 1. Sagittal T2-weighted MR images obtained approximately 3.5 months prior to surgery (left) and immediately prior to surgery (right), showing development of kyphosis.

according to clinically meaningful cutoffs, such that a reasonable sample size would exist in each category, then analyzed as categorical variables. For our secondary outcome, we assessed whether VAS scores differed preoperatively versus postoperatively via 2 -sample paired Student t-test. All statistical analyses were performed using SAS software (version 9.3, SAS Institute).
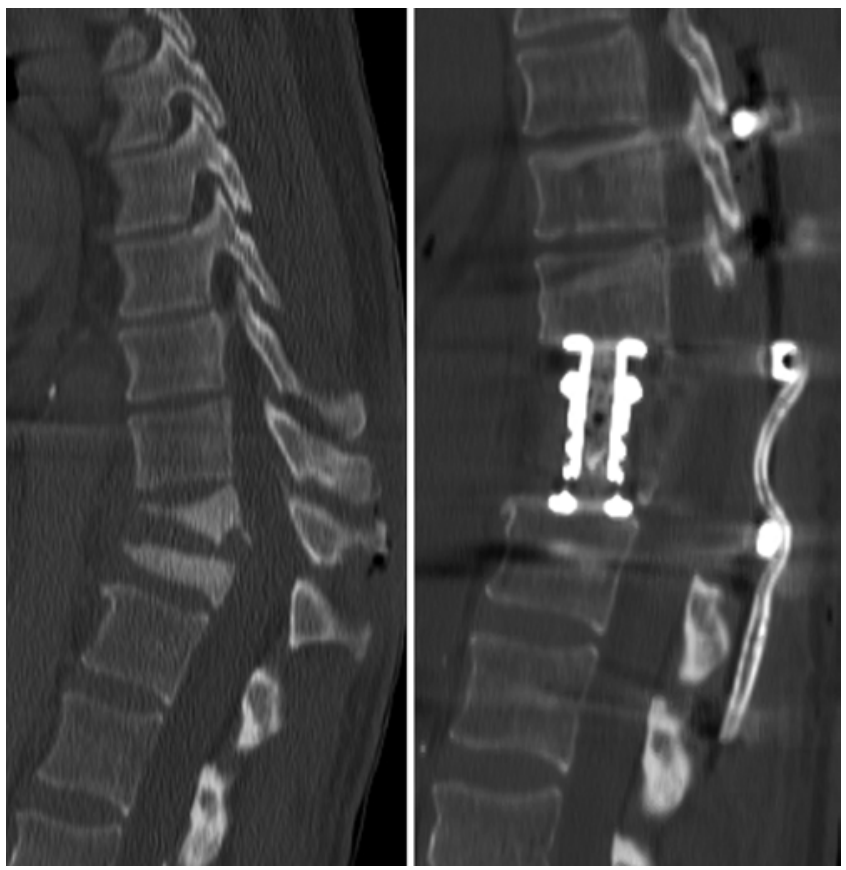

FIG. 2. Sagittal reformatted CT scans showing focal kyphosis due to bone destruction preoperatively (left) and after 2-level corpectomy (right). 


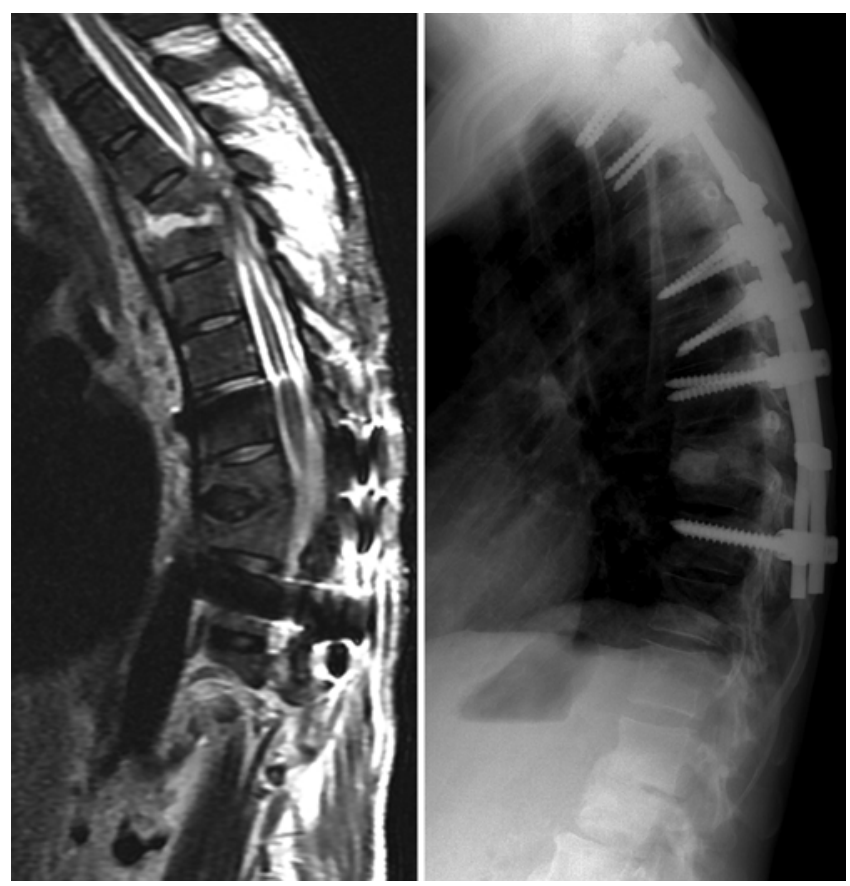

FIG. 3. Sagittal T2-weighted MR image of a patient with a history of prior T8-11 fusion who presented with T4-5 infection with focal kyphosis (left), and postoperative sagittal thoracic radiograph after debridement and extension of fusion to T-2 (right).

\section{Results}

\section{Patient Population}

This study included a total of 48 adult patients with a surgically treated spinal infection. Baseline characteristics of our cohort are described in Table 1. The most common spinal infections were osteomyelitis and discitis (31\%); osteomyelitis, discitis, and SEA (27\%); SEA only (15\%); and osteomyelitis only (13\%). Most infections were caused by Staphylococcus aureus (71\%).

\section{Overall Risk and Predictors of Deformity}

The overall risk of developing a deformity in this cohort was $44 \%$ (21/48). Of the 21 patients developing a deformity, 14 involved kyphosis only, 5 involved subluxation only, and 2 involved both kyphosis and subluxation (Table 1).

Table 2 displays our analysis of the association between a variety of factors and developing at least 1 spinal deformity. The only factor found to be significantly associated with deformity was the particular anatomical combination of infection sites $(\mathrm{p}<0.001)$. Demographics, medical history, and other operative details were not significantly associated with deformity.

Because site of infection was associated with deformity, we further analyzed this variable. Table 3 shows the breakdown of type of infection by type of deformity. Then, we determined the association between a given infection and risk for deformity in Table 4. Having SEA was associated with significantly lower odds of deformity compared with not having SEA (odds ratio $0.2,95 \%$ confidence interval $0.05-0.9 ; \mathrm{p}<0.001)$. Neither osteomyelitis compared with no osteomyelitis, nor discitis compared
TABLE 1: Baseline characteristics of patients surgically treated for spinal infection

\begin{tabular}{|c|c|}
\hline Variable & Overall Cohort $(n=48)$ \\
\hline mean age $\pm \mathrm{SD}$ (yrs) & $56 \pm 12$ \\
\hline mean $\mathrm{BMI} \pm \mathrm{SD}\left(\mathrm{kg} / \mathrm{m}^{2}\right)$ & $29 \pm 10$ \\
\hline males (\%) & $29(60)$ \\
\hline \multicolumn{2}{|l|}{ medical history (\%) } \\
\hline osteoarthritis & $8(17)$ \\
\hline osteoporosis & $4(8)$ \\
\hline hypertension & $30(63)$ \\
\hline diabetes mellitus & $15(31)$ \\
\hline coronary artery disease & $9(19)$ \\
\hline congestive heart failure & $2(4)$ \\
\hline mean preop creatinine level $\pm \mathrm{SD}(\mathrm{mg} / \mathrm{dl})$ & $1.5 \pm 1.9$ \\
\hline previous spine surgery (\%) & $14(29)$ \\
\hline \multicolumn{2}{|l|}{ microbe $(\%)$} \\
\hline S. aureus & $34(71)$ \\
\hline other* & $9(19)$ \\
\hline unknown & $5(10)$ \\
\hline \multicolumn{2}{|l|}{ no. spinal levels infected (\%) } \\
\hline 1 & $34(71)$ \\
\hline 2 & $7(15)$ \\
\hline 3 & $5(10)$ \\
\hline 4 & $1(2)$ \\
\hline 9 & $1(2)$ \\
\hline \multicolumn{2}{|l|}{ infection combination (\%) } \\
\hline osteomyelitis only & $6(13)$ \\
\hline discitis only & $3(6)$ \\
\hline SEA only & $7(15)$ \\
\hline osteomyelitis \& discitis & $15(31)$ \\
\hline osteomyelitis \& SEA & $1(2)$ \\
\hline discitis \& SEA & $3(6)$ \\
\hline all 3 & $13(27)$ \\
\hline $\begin{array}{l}\text { mean time btwn diagnosis \& operation } \pm \\
\text { SD (days) }\end{array}$ & $40 \pm 78$ \\
\hline \multicolumn{2}{|l|}{ spinal deformity (\%) } \\
\hline any & $21(44)$ \\
\hline kyphosis only & $14(29)$ \\
\hline subluxation only & $5(10)$ \\
\hline kyphosis \& subluxation & $2(4)$ \\
\hline mean length of stay $\pm S D$ (days) & $26 \pm 20$ \\
\hline $\begin{array}{l}\text { mean follow-up from surgery to last office } \\
\text { visit } \pm S D \text { (mos) }\end{array}$ & $16 \pm 14$ \\
\hline
\end{tabular}

* Other category included the following: Enterococcus faecalis $(n=2)$, Propionibacterium acnes $(n=1)$, Bacteroides $(n=1)$, Escherichia coli $(n=1)$, Blastomyces $(n=1)$, Streptococcus $(n=1)$, and Pseudomonas aeruginosa $(n=2)$.

with no discitis was significantly associated with deformity. In addition, subgroup analysis excluding patients with SEA did not show an association between number of levels affected and development of deformity $(p=0.74)$. 


\section{Srinivasan et al.}

TABLE 2: Predictors of deformity in patients surgically treated for spinal infection

\begin{tabular}{|c|c|c|}
\hline Variable & $\begin{array}{l}\text { No. Deformity/ } \\
\text { Total (\%) }\end{array}$ & p Value \\
\hline age (yrs) & & 0.17 \\
\hline$<50$ & $4 / 16(25)$ & \\
\hline $50-70$ & $12 / 24(50)$ & \\
\hline$\geq 70$ & $5 / 8(63)$ & \\
\hline BMI $\left(\mathrm{kg} / \mathrm{m}^{2}\right)$ & & 0.87 \\
\hline$<25$ & $7 / 17(41)$ & \\
\hline $25-30$ & $8 / 16(50)$ & \\
\hline$\geq 30$ & $6 / 15(40)$ & \\
\hline sex & & 0.32 \\
\hline male & $11 / 29(38)$ & \\
\hline female & $10 / 19(53)$ & \\
\hline \multicolumn{3}{|l|}{ medical history } \\
\hline w/ osteoarthritis & $4 / 8(50)$ & 0.72 \\
\hline w/o osteoarthritis & $17 / 40(43)$ & \\
\hline w/ osteoporosis & $2 / 4(50)$ & $>0.99$ \\
\hline w/o osteoporosis & $19 / 44(43)$ & \\
\hline w/ hypertension & $15 / 30(50)$ & 0.26 \\
\hline w/o hypertension & $6 / 18(33)$ & \\
\hline w/ diabetes mellitus & $6 / 15(40)$ & 0.72 \\
\hline w/o diabetes mellitus & $15 / 33(45)$ & \\
\hline w/ coronary artery disease & $4 / 9(44)$ & $>0.99$ \\
\hline w/o coronary artery disease & $17 / 39(44)$ & \\
\hline w/ congestive heart failure & $1 / 2(50)$ & $>0.99$ \\
\hline w/o congestive heart failure & $20 / 46(43)$ & \\
\hline preoperative creatinine level & & 0.73 \\
\hline$<1.3(\mathrm{mg} / \mathrm{dl})$ & $17 / 37(46)$ & \\
\hline$\geq 1.3(\mathrm{mg} / \mathrm{dl})$ & 4/11 (36) & \\
\hline previous spine surgery & & 0.23 \\
\hline yes & $8 / 14(57)$ & \\
\hline no & 13/34 (38) & \\
\hline microbe & & 0.66 \\
\hline S. aureus & $15 / 34(44)$ & \\
\hline other & $3 / 9(33)$ & \\
\hline unknown & $3 / 5(60)$ & \\
\hline no. spinal levels affected & & 0.62 \\
\hline 1 & $15 / 34(44)$ & \\
\hline 2 & $2 / 7(29)$ & \\
\hline 3 & $3 / 5(60)$ & \\
\hline 4 & $1 / 1(100)$ & \\
\hline 9 & $0 / 1(0)$ & \\
\hline infection combination & & $<0.001$ \\
\hline osteomyelitis only & $4 / 6(67)$ & \\
\hline discitis only & $3 / 3(100)$ & \\
\hline SEA only & $0 / 7(0)$ & \\
\hline osteomyelitis \& discitis & $11 / 15(73)$ & \\
\hline osteomyelitis \& SEA & $0 / 1(0)$ & \\
\hline
\end{tabular}

TABLE 2: Predictors of deformity in patients surgically treated for spinal infection (continued)

\begin{tabular}{lcc}
\hline \multicolumn{1}{c}{ Variable } & $\begin{array}{c}\text { No. Deformity/ } \\
\text { Total (\%) }\end{array}$ & p Value* \\
\hline $\begin{array}{l}\text { infection combination (continued) } \\
\text { discitis \& SEA }\end{array}$ & $1 / 3(33)$ & $<0.001$ \\
$\quad$ all 3 & $2 / 13(15)$ & \\
days btwn diagnosis \& operation & & 0.29 \\
$\quad<30$ & $9 / 26(35)$ & \\
$30-60$ & $4 / 9(44)$ & \\
$\geq 60$ & $8 / 13(62)$ & \\
length of stay in days & & 0.28 \\
$\quad<14$ & $5 / 15(33)$ & \\
$14-30$ & $11 / 19(58)$ & \\
$\geq 30$ & $5 / 14(36)$ & \\
\hline
\end{tabular}

* The $p$ values were obtained using either chi-square or Fisher exact tests. Significant values are in boldface.

\section{Pain Outcomes}

The mean preoperative VAS score was $4.9 \pm 3.4$. A mean VAS score of $3.1 \pm 2.7$ was recorded at the final follow-up visit (after a mean of $16 \pm 14$ months postoperatively). Patient VAS scores improved significantly on average by a mean of $1.7 \pm 2.7(\mathrm{p}<0.001)$.

\section{Discussion}

Bacterial infections of the spine, including vertebral osteomyelitis, discitis, and epidural abscesses, although relatively infrequent, ${ }^{3,13}$ comprise an important clinical entity with a high risk for death and potentially serious neurological disability., 40,18 One series identified an $11 \%$ risk of death and $31 \%$ risk of clinically significant residual neurological deficits such as paralysis, neurogenic bladder, or intractable pain following a spinal infection. ${ }^{10}$ Aside from feared neurological deficits, another potential consequence of infection may be bone destruction caused by the infection, resulting in spinal deformity. However, neither the risk of deformity nor risk factors associated with development of deformity has thus far been clearly elucidated in the literature.

Spinal infections may lead to deformity via a number of mechanisms. Spinal infections occur frequently due to hematogenous dissemination, often secondary to bacteremia due to $S$. aureus. ${ }^{2,6}$ Seeding of the spinal cord often occurs from arterial vasculature to the metaphysis of individual vertebrae, or less frequently by the Batson's plexus or the deep pelvic venous system. ${ }^{2,5,17}$ This vascular spread of bacterial inoculation or pus can create an increase in intraosseous pressure that impedes blood flow to the vertebrae and intervertebral discs..$^{8,15,16}$ This process may lead to ischemic necrosis of the vertebral body and disc, compromising structural integrity and causing spinal deformity via vertebral body destruction. In another mechanism, bacterial agents such as $S$. aureus produce destructive enzymes - most importantly, hyaluronidase- 


\section{Risk of deformity from spinal infection}

TABLE 3: Infection type/combination dichotomized by deformity type

\begin{tabular}{lccccc}
\hline \multirow{2}{*}{ Infection } & \multicolumn{5}{c}{ Deformity } \\
\cline { 2 - 5 } & None & Kyphosis & Subluxation & Both & Total \\
\hline osteomyelitis only & 2 & 2 & 2 & 0 & 6 \\
discitis only & 0 & 2 & 0 & 1 & 3 \\
SEA only & 7 & 0 & 0 & 0 & 7 \\
osteomyelitis \& discitis & 4 & 8 & 2 & 1 & 15 \\
osteomyelitis \& SEA & 1 & 0 & 0 & 0 & 1 \\
discitis \& SEA & 2 & 1 & 0 & 0 & 13 \\
all 3 & 11 & 1 & 1 & 2 & 48 \\
total & 27 & 14 & 5 & & \\
\hline
\end{tabular}

that enhance the bacteria's ability to invade connective tissue, such as the annulus fibrosis. This proteolytic enzyme causes a breakdown of the structural fibers of the annulus fibrosis, leading to biomechanical instability and deformity characterized by prespinous disc extrusion. ${ }^{7}$

In this series, 21 (44\%) of 48 patients diagnosed with a spinal infection subsequently developed a spinal deformity. The only factor found to be significantly associated with deformity was the anatomical spaces involved. In particular, the presence of SEA was associated with significantly lower odds for deformity than infections in other locations. This observation is somewhat intuitive and pathophysiologically plausible, given that an infection not affecting the bone or disc may be less likely to cause a change in spinal alignment. No other analyzed covariate, including age, BMI, time from initial diagnosis to surgery, and comorbidities, were associated with development of deformity.

Our study contains a number of limitations. First, our sample size may have led to a high Type II error; thus, we did not detect significant predictors that may have been found in a larger cohort. Given the uncommon presentation of infection, series reporting infections often suffer from small sample sizes, but we did include enough patients to detect 1 important significant association between infection type and deformity. Second, as a retrospective study, our analysis may be prone to bias and confounding, but this was an exploratory study and may serve as the basis for future larger series with multivariable adjustment. And third, VAS scores are prone to recall bias and can be influenced by complex factors unrelated to surgery. Nonetheless, they do provide an important patient-centered outcome variable.

\section{Conclusions}

Overall, $44 \%$ of patients in this cohort developed at least 1 deformity, predominantly kyphosis. The only variable significantly associated with deformity was infection location, and patients with SEA demonstrated lower odds of developing a deformity compared with patients without SEA. Other analyzed variables, including age, BMI, time from initial diagnosis to surgery, and comorbidities, were not found to be associated with development of deformity. Surgical intervention resulted in pain improvement.

\section{Disclosure}

Dr. La Marca has served as a consultant to Globus and Biomet. Dr. Park has served as a consultant to Globus, Medtronic, and Biomet, and has received royalties from Globus.

Author contributions to the study and manuscript preparation include the following. Conception and design: Park, La Marca. Acquisition of data: all authors. Analysis and interpretation of data: Srinivasan, Terman, Himedan, Dugo. Drafting the article: Srinivasan, Terman, Himedan, Dugo. Critically revising the article: Park. Reviewed submitted version of manuscript: all authors. Approved the final version of the manuscript on behalf of all authors: Park. Study supervision: Park, La Marca.

\section{References}

1. Arnold PM, Baek PN, Bernardi RJ, Luck EA, Larson SJ: Surgical management of nontuberculous thoracic and lumbar vertebral osteomyelitis: report of 33 cases. Surg Neurol 47:551-561, 1997

2. Carragee EJ: Pyogenic vertebral osteomyelitis. J Bone Joint Surg Am 79:874-880, 1997

3. Chen WH, Jiang LS, Dai LY: Surgical treatment of pyogenic vertebral osteomyelitis with spinal instrumentation. Eur Spine J 16:1307-1316, 2007

TABLE 4: Association between infection type and risk for deformity

\begin{tabular}{lccc}
\hline \multicolumn{1}{c}{ Infection } & No. Deformity/Total $(\%)$ & OR $(95 \% \mathrm{Cl})$ & $\mathrm{p} \mathrm{Value}^{*}$ \\
\hline w/ osteomyelitis & $17 / 35(49)$ & $2.1(0.6-8.2)$ & 0.27 \\
w/o osteomyelitis & $4 / 13(31)$ & & 0.17 \\
w/ discitis & $17 / 34(50)$ & $2.5(0.7-10)$ & \\
w/o discitis & $4 / 14(29)$ & & $<0.001$ \\
w/ SEA & $3 / 24(13)$ & $0.2(0.05-0.9)$ & \\
w/o SEA & $18 / 24(75)$ & & \\
\hline
\end{tabular}

* Values obtained using the chi-square test. Significant values are in boldface. $\mathrm{Cl}=$ confidence interval; $\mathrm{OR}=$ odds ratio. 


\section{Srinivasan et al.}

4. Darouiche RO: Spinal epidural abscess. N Engl J Med 355: 2012-2020, 2006

5. Eismont FJ, Bohlman HH, Soni PL, Goldberg VM, Freehafer AA: Pyogenic and fungal vertebral osteomyelitis with paralysis. J Bone Joint Surg Am 65:19-29, 1983

6. Hsieh PC, Wienecke RJ, O'Shaughnessy BA, Koski TR, Ondra SL: Surgical strategies for vertebral osteomyelitis and epidural abscess. Neurosurg Focus 17(6):E4, 2004

7. Jinkins JR, Bazan C III, Xiong L: MR of disc protrusion engendered by infectious spondylitis. J Comput Assist Tomogr 20:715-718, 1996

8. Lew DP, Waldvogel FA: Osteomyelitis. Lancet 364:369-379, 2004

9. McHenry MC, Duchesneau PM, Keys TF, Rehm SJ, Boumphrey FR: Vertebral osteomyelitis presenting as spinal compression fracture. Six patients with underlying osteoporosis. Arch Intern Med 148:417-423, 1988

10. McHenry MC, Easley KA, Locker GA: Vertebral osteomyelitis: long-term outcome for 253 patients from 7 Cleveland-area hospitals. Clin Infect Dis 34:1342-1350, 2002

11. Osenbach RK, Hitchon PW, Menezes AH: Diagnosis and management of pyogenic vertebral osteomyelitis in adults. Surg Neurol 33:266-275, 1990

12. Rezai AR, Woo HH, Errico TJ, Cooper PR: Contemporary management of spinal osteomyelitis. Neurosurgery 44:10181026, 1999

13. Rigamonti D, Liem L, Sampath P, Knoller N, Namaguchi Y, Schreibman DL, et al: Spinal epidural abscess: contemporary trends in etiology, evaluation, and management. Surg Neurol 52:189-197, 1999

14. Vandenbroucke JP, von Elm E, Altman DG, Gøtzsche PC, Mulrow $\mathrm{CD}$, Pocock SJ, et al: Strengthening the Reporting of Observational Studies in Epidemiology (STROBE): explanation and elaboration. Ann Intern Med 147:W163-W194, 2007

15. Waldvogel FA, Medoff G, Swartz MN: Osteomyelitis: a review of clinical features, therapeutic considerations and unusual aspects. 3. Osteomyelitis associated with vascular insufficiency. N Engl J Med 282:316-322, 1970

16. Waldvogel FA, Papageorgiou PS: Osteomyelitis: the past decade. N Engl J Med 303:360-370, 1980

17. Weinstein JN, Lurie JD, Tosteson TD, Hanscom B, Tosteson AN, Blood EA, et al: Surgical versus nonsurgical treatment for lumbar degenerative spondylolisthesis. N Engl J Med 356: 2257-2270, 2007

18. Zimmerli W: Clinical practice. Vertebral osteomyelitis. N Engl J Med 362:1022-1029, 2010

Manuscript submitted April 15, 2014.

Accepted June 11, 2014.

Please include this information when citing this paper: DOI: 10.3171/2014.6.FOCUS14143.

Address correspondence to: Paul Park, M.D., Department of Neurosurgery, University of Michigan, 1500 E. Medical Center Dr., Rm. 3552 TC, Ann Arbor, MI 48109-5338. email: ppark@umich.edu. 